\title{
A prospective comparison of transvaginal, transabdominal ultrasound and diagnostic curettage in the evaluation of endometrial pathology in Erbil

\section{Abstract}

Background and objective: Abnormal uterine bleeding due to endometrial abnormalities is a common diagnostic challenge facing the radiologist and referring gynecologist. This study was aimed to compare the diagnostic performance of transvaginal ultrasound, transabdominal ultrasound and diagnostic curettage in the detection of endometrial pathologies in symptomatic women.

Methods: A prospective comparison study of transvaginal ultrasound, transabdominal ultrasound and diagnostic curettage was conducted for evaluation of endometrial pathology in Maternity Teaching Hospital, Erbil city,Kurdistan region of Iraq, from September $13^{\text {th }}$, 2013 to September $14^{\text {th }}, 2014$. The study included 100 women presenting with abnormal uterine bleeding. Ultrasound findings were compared with histopathological results. The statistical package for the social sciences (version 17) was used for data entry and analysis.

Results: The mean age ( \pm SD) of sample size was $47 \pm 8.57$ years. The mean parity was $5.17 \pm 2.71$. The highest percentages of women were in age group $40-59$ years $(73 \%)$. Of the total sample, more than half $(52 \%)$ had regular cycles, $25 \%$ had irregular cycles and $23 \%$ were in menopausal state. In more than half of cases the indication for ultrasounds was for menorrhagia, followed by postmenapousal and intermenstrual bleeding $(23 \%$ and $21 \%$, respectively). Of 98 women (2 women were excluded from analysis), $19 \%$ had atrophic endometrium and $67 \%$ had endometrial hyperplasia in histopathological finding. Transvaginal ultrasound sensitivity and specificity were $100 \%$ and $92.9 \%$, respectively while transabdominal ultrasound sensitivity and specificity were $92.8 \%$ and $65 \%$, respectively.

Conclusion: Transvaginal ultrasound scanning is an excellent tool for the determination of whether further investigation with histopathological examination of endometrial biopsy is necessary or not for women presented with abnormal uterine bleeding.

Keywords: Transvaginal, Transabdominal ultrasound, Endometrial pathology.

\section{Introduction}

Abnormal uterine bleeding due to endometrial abnormalities is a common diagnostic challenge facing the radiologist and referring gynecologist. The cycle length, the volume and duration of bleeding remain fairly constant for a woman throughout her reproductive years. After menopause, bleeding ceases completely. ${ }^{1,2}$ The endometrium demonstrates a wide spectrum of normal and pathologic appearances throughout menarche as well as during the prepubertal and postmenopausal years. The appearance of the endometrium is related to multiple factors, including patient's age, stage in the menstrual cycle, and whether she has undergone hormonal replacement therapy. Abnormal vaginal bleeding may include noncyclical, excessive, or prolong bleeding in premenopausal or any bleeding in postmenopausal woman. ${ }^{3} \quad$ Ultrasound plays a significant role in evaluation of endometrium, it is inexpensive, non-invasive

*Department of surgery, college of medicine, Hawler Medical University, Erbil, Iraq.

$* *$ Department of obstetrics and gynaecology, college of medicine, Hawler Medical University, Erbil, Iraq.

$* * *$ Department of obstetrics and gynaecology, Maternity Teaching Hospital, Erbil, Iraq. 
and performed in outpatient clinics with minimal inconvenience to the patient. Some indications of pelvic ultrasound include infertility, heavy or irregular vaginal bleeding, postmenopausal bleeding and pelvic pain. ${ }^{4,5}$ The transvaginal ultrasound (TVS) is an internal ultrasound. It is used for indirect visualization of the endometrium. It involves scanning with the ultrasound probe lying in the vagina. It usually produces better and clearer images of the female pelvic organs, because the ultrasound probe lies closer to these structures. $^{6}$ Transabdominal ultrasound (TAS) is used to assess the female pelvic organs and scanning through lower abdomen, it provides an overview of the pelvis rather than detailed images. It is particularly helpful for the examination of large pelvic masses extending into the abdomen, which are not always well viewed with TVS. ${ }^{7,8}$ Dilatation and curettage is the traditional technique for obtaining samples of endometrium for pathological examination. The endometrium is sampled when pathology is suspected; this may be when the patient experiences a change in normal pattern of menstrual bleeding. ${ }^{6,9}$ This study was carried out to determine the sensitivity and specificity of both TAS and TVS for detection of endometrial abnormality through comparison with the histopathological results of various age groups presenting with abnormal uterine bleeding.

\section{Methods}

A prospective comparison study of TVS, TAS and diagnostic curettage (D\&C) in the evaluation of endometrial pathology was conducted in Maternity Teaching Hospital, Erbil city, Kurdistan region, Iraq from September 2013 to September 2014. A sample of 100 symptomatic women of different age groups, during reproductive premenopausal and postmenopausal period presented to the gynecology outpatient clinic with abnormal uterine bleeding who agreed to participate in the study were included. Women with submucus fibroid, cervical and vaginal pathology, endometritis and haemostatic disorders and those who refused to participate in the study were excluded. The study was approved by the Research Ethics Committee at the College of Medicine of Hawler Medical University. Abdominal, vaginal and D\&C procedures were explained to the patients and informed verbal and written consents were taken from each participant. Pelvic ultrasound was done for them in early proliferative phase followed by endometrial biopsy for histopathological examination using D\&C. All ultrasound procedures were conducted by the same sonologist in a private clinic. The ultrasound machine used was General Electric (GE), Voluson 6 Machine. TAS ultrasound was done first, in which the patient was asked to drink several glasses of water or any type of juice one hour before the procedure to have full bladder. Examination was done with a smooth, hand-held curved transducer $\mathrm{C} 4 \mathrm{MHZ}$. The abdomen was exposed and clear gel was applied to the skin and the transducer was moved gently across the abdomen with a sliding and rotating action, a long axis of endometrium obtained and endometrium measured in thickest part from echogenic to echogenic border. The adjacent hypo echoic myometrium was not included. The endometrium was also analyzed for any focal abnormality, echogenicity, and the presence of fluid or masses in the cavity. Then TVS was performed after asking the women to empty her bladder. undress from the waist down, and lie in a dorsal position on the examination couch. A sheet was provided to cover her. She was asked to bend her legs, then vaginal transducer E8 MHZ was used. The probe covered with a plastic or latex sheath and lubricated with gel and the tip of the transducer was inserted into vagina. The endometrium was analyzed for thickness, focal abnormality, echogenicity, and presence of fluid or masses in the cavity. The thickest part of the 
endometrium was obtained and measured perpendicular to its longitudinal plane in the anteroposterior diameter from echogenic to echogenic border. The adjacent hypo echoic myometrium and fluid in the cavity was excluded. In the presence of endometrial fluid, the measurement of the two separate layers of the endometrium, excluding the fluid, were added to determine the endometrial thickness. Then after proper scanning the transducer was withdrawn gently toward fornix to avoid discomfort to the patient, all transvaginal probes have been cleaned and sterilized. D\&C was conducted in the theater under general anesthesia according to hospitals regulations. All ultrasound scanning were conducted in the early follicular phase of the menstrual cycle where endometrial thickness regarded normal if it was $5-7 \mathrm{~mm}$ and more than $7 \mathrm{~mm}$ this thickness was regarded as increased thickness. The postmenopausal endometrium thickness was regarded normal if it was $5 \mathrm{~mm}$ or less. ${ }^{8,9}$ Endometrium regularity was assessed and any focal thickness or masses in the endometrium were identified.

Statistical analysis: The statistical package for the social sciences (version 17) was used for data entry and analysis. Two approaches were used; descriptive and analytic. The descriptive approach included calculation of frequencies and percentages, while, in the second approach; two by two table was used to calculate sensitivity and specificity between TAS findings, and TVS findings with histopathological findings. McNemar test was used to show whether the differences between the test results are significant or not. $P$ value of $\leq 0.05$ was regarded as statistically significant.

\section{Results}

Hundred women presented with abnormal uterine bleeding were included in the current study (26 were post-menopausal and 74 were in pre-menopausal state), two women were excluded from analysis regarding sensitivity and specificity because endometrial samples from D\&C procedure were not sufficient for histopathological examination. Of the total sample (100); the mean ( \pm SD) age was $47 \pm 8.57$, ranged from $30-70$ years (reproductive, premenopausal and post-menopausal age group) and the mean $( \pm \mathrm{SD})$ parity was $5.17 \pm 2.71$, ranged from $0-13$. The highest percentage of women was in age group 40-59 year (73\%). Of total sample; $50 \%$ had regular cycle, $24 \%$ had irregular cycle and $26 \%$ had no cycle. In more than half of cases the indication for ultrasound was menorrhagia, followed by postmenapousal and intermenstrual bleeding $(23 \%$ and $21 \%$, respectively) as shown in Table 1.

Table 1: Frequency of indications for ultrasound.

\begin{tabular}{lll}
\hline Indications & No. & $\%$ \\
\hline Menorrhagia & 51 & 51.0 \\
Intermenstrual bleeding & 21 & 21.0 \\
Poly menorrhea & 5 & 5.0 \\
Postmenapousal bleeding & 23 & 23.0 \\
Total & 100 & 100.0 \\
\hline
\end{tabular}


Of total 74 women of reproductive and premenopausal age; $56(75.7 \%)$ cases had endometrial hyperplasia, and only 8 $(10.8 \%)$ cases had endometrial polyp, while among the total 26 women of postmenopausal age, only 11 (42.3) cases had endometrial hyperplasia. Details of other histopathological findings are illustrated in Table 2. Table 3 shows the histopathological finding versus transvaginal and transabdominal ultra sound findings.

Table 2: Frequency of histopathological results among study sample.

\begin{tabular}{lllllll}
\hline & \multicolumn{2}{l}{ Age } & & & \multicolumn{2}{l}{} \\
Histopathological diagnosis & \multicolumn{2}{l}{$\begin{array}{l}\text { Reproductive } \\
\text { \&premenopausal }\end{array}$} & \multicolumn{2}{l}{ Post-menopausal } & Total \\
& No & $\%$ & No. & $\%$ & No. & $\%$ \\
\hline Atrophic endometrium & $6^{*}$ & 8.1 & 13 & 50 & 19 & 19 \\
Endometrial hyperplasia & 56 & 75.7 & 11 & 42.3 & 67 & 67 \\
Endometrial polyp & 8 & 10.81 & 1 & 3.84 & 9 & 9 \\
Endometrial carcinoma & 0 & 0.0 & 1 & 3.84 & 1 & 2 \\
Retained pieces of conception & 2 & 2.7 & 0 & 0.00 & 2 & 2 \\
Sample not sufficient for evalua- & 2 & 2.7 & 0 & 0.00 & 2 & 2 \\
tion & 74 & 100 & 26 & 100 & 100 & 100 \\
\hline Total & & & & & & \\
\hline
\end{tabular}

${ }^{*}$ All in perimenopausal state.

Table 3: Histopathological finding versus transvaginal and transabdominal ultrasound findings among study sample.

\begin{tabular}{lllllll}
\hline \multirow{2}{*}{ Diagnosis } & \multicolumn{3}{c}{ Histopathology } & \multicolumn{2}{c}{ Transvaginal } & \multicolumn{2}{c}{ Tranabdominal } \\
& No. & $\%$ & No. & $\%$ & No. & $\%$ \\
\hline Atrophic endometrium & 19 & 19.3 & $18^{*}$ & 18.4 & $14^{*}$ & 14.3 \\
Endometrium hyperplasia & 67 & 68.3 & 69 & 70.4 & 80 & 81.6 \\
Endometrial polyp & 9 & 9.10 & $6^{* *}$ & 6.10 & $2^{* *}$ & 2.04 \\
Endometrial carcinoma & 1 & 1.02 & $1^{\#}$ & 1.02 & 0 & 0.00 \\
Retained piece of conception & 2 & 2.04 & 4 & 4.08 & 2 & 2.04 \\
Total & 98 & 100 & 98 & 100 & 98 & 100 \\
\hline *Normal looking endometrium by ultrasound according to the age group. & & & & \\
** Mass or focal thickening, suggestive of polyp. \\
\# Irregular mass.
\end{tabular}


This study revealed that the sensitivity and specificity of TAS of $100 \%$ and $68.4 \%$, respectively $(P<0.001)$. The sensitivity and specificity of TVS was $100 \%$ and $94.7 \%$, respectively $(P=1)$ as shown in Table 4 and 5.

\section{Discussion}

The current study revealed that the most common types of abnormal uterine bleeding in the study groups was menorrhagia $(51 \%$ of women. Pyari et al

Table 4: Sensitivity and specificity of transabdominal US among study sample.

\begin{tabular}{lllll}
\hline Transabdominal US & \multicolumn{2}{l}{ Histopathological } & Total & $\boldsymbol{P}$ value \\
& Abnormal & Normal & 85 & \\
\hline \multirow{2}{*}{ Abnormal } & 79 & 6 & $100.0 \%$ & \\
& $92.9 \%$ & $7.1 \%$ & $86.7 \%$ & \\
& $100.0 \%$ & $31.6 \%$ & 13 & $<0.001$ \\
Normal & 0.0 & 13 & $100.0 \%$ & \\
& $0.0 \%$ & $100.0 \%$ & $13.3 \%$ & \\
& $0.0 \%$ & $68.4 \%$ & 98 & \\
Total & 79 & 19 & $100.0 \%$ & \\
& $80.6 \%$ & $19.4 \%$ & $100.0 \%$ & \\
\hline
\end{tabular}

Table 5: Sensitivity and specificity of transvaginal US among study sample.

\begin{tabular}{lllll}
\hline Transvaginal US & $\begin{array}{l}\text { Histopathological } \\
\text { Abnormal }\end{array}$ & Normal & Total & $\boldsymbol{P}$ value \\
\hline Abnormal & 79 & 1 & 80 & \\
& $98.8 \%$ & $1.3 \%$ & $100.0 \%$ & \\
& $100.0 \%$ & $5.3 \%$ & $81.6 \%$ & 1 \\
Normal & 0.0 & 18 & 18 & \\
& $0.0 \%$ & $100.0 \%$ & $100.0 \%$ & $18.4 \%$ \\
& $0.0 \%$ & $94.7 \%$ & 98 & \\
Total & 79 & 19 & $100.0 \%$ & \\
& $80.6 \%$ & $19.4 \%$ & $100.0 \%$ & \\
\hline
\end{tabular}

Abnormal= Abnormal endometrium (endometrial hyperplasia, endometrial polyp, endometrial carcinoma and retained piece of conception)

Normal=Normal endometrium 
studied 70 patients, of those 50 having abnormal uterine bleedingand the common symptoms in patients with abnormal uterine bleeding were menorrhagia (40\%) followed by metrorrhagia (18\%), menometrorrhagia $(14 \%)$, and polymenorrhea $(14 \%) .^{10}$ The current study showed that endometrial hyperplasia, was the commonest finding by histopathology, also commonest finding by ultrasound and also the commonest cause of abnormal uterine bleeding in premenapausal age group. Similar data was published by Anwer et $\mathrm{al}^{11}{ }^{11}$ where endometrial hyperplasia was diagnosed by ultrasound with the appearance of well defined, thick and highly reflective layer occupying the whole of the endometrial cavity. Three cases in the histopathological examination revealed atypical hyperplasia; all were in the postmenopausal age groups. Depending on the endometrial thickness in the post-menopausal women, any endometrial thickness more than five was regarded to be abnormal in post-menopausal women, dilatation and curettage is recommended for these women with abnormal uterine bleeding as the risk of malignancy is increased up to $44.5 \%{ }^{12}$ In the current study the atrophic endometrium among post-menopausal women was the second most common histopathological and ultrasound finding mainly in $11 \%$. This finding is near to finding reported in a study done by Opolskiene et al $(13 \%){ }^{13}$ Of 98 cases, the histopathological findings showed endometrial polyp in 9 cases presented with abnormal uterine bleeding (9.1\%), which was more common in premenopausal and reproductive age group. Six cases $(6.1 \%)$ were diagnosed by TVS to be endometrial polyp, while TAS revealed the polyp in only 2 cases $(2.04 \%)$. Although endometrial polyps may be visualized at TVS as nonspecific endometrial thickening, they may also be identified as focal masses within the endometrial canal, a stalk to the polyp may be either thin or broad based,a feeding vessel may be seen extending to the polyp on Colour Doppler imaging or cystic spaces corresponding to dilated glands filled with proteinaceous fluid which may be seen within the polyp and is considered a relatively characteristic feature, or may appear as just diffusely thickened endometrium, without visualization of a discrete mass (mimicking endometrial hyperplasia). ${ }^{14}$ The sensitivity of TVS was $100 \%$ and specificity $94.7 \%$ for detecting endometrial pathologies; this is in agreement with other studies, ${ }^{12}$ while sensitivity and specificity of TAS was $100 \%$ and $68.4 \%$.

\section{Conclusion}

Both TVS and TAS were highly sensitive for detecting endometrial hyperplasia while TVS is more sensitive and specific in detecting focal masses or polyps within endometrial cavity because of clearer and better images due to closer ultrasound probe to internal structures. This study also concluded that transvaginal sonographic scanning is an excellent tool for the determination of whether curettage or endometrial biopsy is necessary or not.

\section{Conflicts of interest}

The authors report no conflicts of interest.

\section{References}

1. Ascher S, Imaoka I, Lage J. Tamoxifen-induced uterine abnormalities: the role of imaging. Radiology 2000; 214: 29-38.

2. Bree R, Bowerman R, Bohm-Velez M, Benson C, DeDreu S, Punch M. US evaluation of the uterus in patients with postmenopausal bleeding: A positive effect on diagnostic decision making. Radiology 2000; 216: 260-4.

3. Dangal G. A study of endometrium of patients with abnormal uterine bleeding at Chitwan valley. Kathmandu Univ Med J 2003;1: 110-2.

4. Dimitraki $M$, Tsikouras $P$, Bouchlariotou $S$, Dafopoulos A, Liberis V, Maroulis G, TeichmannAT.Clinical evaluation of women with PMB. Is it always necessary an endometrial ArchGynecolObstet 2011; 283(2): 261-6.

5. Ely J, Kennedy C, Clark E, Bowdler N. Abnormal Uterine Bleeding: A Management Algorithm. J AmerBoardFam Med 2006; 19: 590-602.

6. Fleischer A. Sonographic assessment of endometrial disorders. SeminUltrasoundCT MR 1999 20; 259-66. 
7. Katz V. Benign gynaecologic lesions: vulva, vagina, cervix, uterus, oviduct, ovary, ultrasound imaging of pelvic structures. Comprehensive Gynaecology. 6th ed. Philadelphia: Elsevier; 2012.

8. Nalaboff K, Pellerito J, Ben-Levi E. Imaging the endometrium: disease and normal variants. Radio graphics 2001; 21: 1409-42.

9. Nutis M, Garcia K, Nuwayhid B, Mulla Z, EIMasri W.Use of ultrasonographic cut point for diagnosing endometrial pathology in postmenopausal women with multiple risk factors for endometrial cancer. J Reprod Med 2008; 53 (10): 75

10. Pyari S, Rekha P, Srivastava M, Goel M, Pandey. A comparative diagnostic evaluation of hysteroscopy, transvaginal ultrasonography and histopathological examination in cases of abnormal uterine bleeding. J Obstet Gynaecol India 2006; 56: 240-3

11. Anwer M,Imdad S, Jamal Q. Histopathological Correlation of endometrial Curettage with abnormal uterine bleeding Pattern. J Surg Pak $2004 ; 9: 21-4$

12. Gull B, Karlsson B, Milsom I, Granberg S. Can ultrasound replace dilation and curettage? A longitudinal evaluation of postmenopausal bleeding and transvaginal sonographic measurement of the endometrium as predictors of endometrial cancer. Am J Obstet Gynecol 2003; 188(2): 401-8.

13. Opolskiene $G$, Sladkevicius $P$, Valentin $L$. Ultrasound assessment of endometrial morphology and vascularity to predict endometrial malignancy in women with postmenopausal bleeding and sonographic endometrial thickness $\geq 4.5 \mathrm{~mm}$. Ultrasound Obstet Gynecol 2007; 30(3): 332-40.

14. Hulka CA, Hall D, Mccarthy K, Simeone J. Endometrial polyps, hyperplasia, and carcinoma in postmenopausal women: differentiation with endovaginal sonography. Radiology 1994; 191 (3):755-8. 